\title{
Análise Regional de Chuvas Intensas para a Bacia do Rio Doce
}

\author{
Ana Esmeria Lacerda Valverde \\ Universidade Federal de Viçosa - UFV - Viçosa - MG \\ valverde@alunos.ufv.br
}

Demetrius David da Silva, Fernando Falco Pruski, Hélio Garcia Leite e Viviane dos Santos Brandão

david@ufv.br,ffpruski@ufv.br,.hgleite@ufv.brevbrandao@buynet.com.br

Recebido: 30/08/02 - revisado: 07/03/03 - aceito: 25/06/03

\section{RESUMO}

O presente trabalho apresenta a aplicação da metodologia de regionalização, proposta por HOSKING e W ALLIS (1997), para a análise de freqüência regional de chuvas intensas, na bacia do rio Doce. Foram analisadas as séries históricas de precipitação de 31 estações pluviográficas, visando a identificação das regiões homogêneas, tendo-se utilizado o método dos momentos-L para estimar os parâmetros das distribuiç̃̃es de probabilidade com melhor aderência aos dados de intensidades máximas médias de precipitação para cada duração.

Com os valores de intensidade máxima média de precipitação correspondentes aos diversos períodos de retorno e durações estudados, obtidos após o ajuste da distribuição de probabilidade com melhor aderência aos dados observados, foram estimados os parâmetros de um modelo nãolinear e definidas as equações de intensidade, duração e freqüência para cada uma das cinco regiões homogêneas.

As distribuições dos resíduos para as equações de intensidade, duração e freqüência individualizadas considerando durações de 10 a 60 minutos e de 60 a 1440 minutos apresentaram comportamento melhor do que quando o periodo considerado foi de 10 a 1440 minutos.

O método de regionalização proposto permite estimar a intensidade máxima média de precipitação para uma duração e período de retorno específicos em qualquer local da bacia, bastando para isto conbecer a precipitação média anual do local considerado.

Palavras-chaves: regiões homogêneas, distribuição de probabilidade e modelo não linear.

\section{INTRODUÇÃO}

$\mathrm{Na}$ análise de freqüência de eventos extremos, geralmente as séries históricas anuais de chuvas intensas representam as variáveis aleatórias. Essas variáveis são ajustadas por meio de uma função densidade de probabilidade e, em seguida, os quantis de precipitações máximas, para um dado período de retorno, podem ser estimados. Contudo, nesse estudo, quase sempre surgem problemas como a inexistência ou escassez de informações ao longo do tempo ou em dadas regiões da bacia hidrográfica.

Uma alternativa para contornar o problema de escassez de dados é a regionalização de chuvas intensas, que consiste na transferência de informações de um local para outro, dentro de uma área com comportamento hidrológico semelhante.

A aplicação de métodos de regionalização tem como vantagem permitir extrair informações dos dados puntais existentes em uma região, extrapolando-os espacialmente e possibilitando uma melhor estimativa dos parâmetros e quantis de uma distribuição de probabilidade teórica.

Tendo em vista a importância do estudo regional de chuvas intensas, o presente trabalho teve como objeti- vos: identificar regiões homogêneas, em relação às chuvas intensas, para a bacia do rio Doce; ajustar modelos teóricos de distribuição de probabilidade às séries históricas regionais de precipitações máximas adimensionalizadas, com base no método "index-flood"; e estabelecer equações de intensidade, duração e freqüência regionais para a referida bacia.

\section{METODOLOGIA DOS MOMENTOS-L PARA ANÁLISE DE FREQÜÊNCIA REGIONAL}

Uma das técnicas usadas atualmente para análise de freqüência regional foi desenvolvida por Hosking e Wallis (1997) e utiliza os momentos-L na definição de regiões homogêneas e da função de distribuição de probabilidade regional (Pinheiro e Naghettini, 1998).

Os momentos-L são derivados dos Momentos Ponderados por Probabilidade (MPP), os quais foram introduzidos por Greenwood et al. em 1979, citados por Pinheiro e Naghettini (1998). Segundo Hosking e Wallis (1997) a estimativa dos momentos-L, a partir de uma amostra finita de tamanho $n$, inicia-se com a ordenação de seus elementos constituintes em ordem crescente, ou seja, $\mathrm{x}_{1: \mathrm{n}} \leq \mathrm{x}_{2: \mathrm{n}} \leq \ldots \leq \mathrm{x}_{\mathrm{n}: \mathrm{n}}$. Os estimadores não-viesados dos 
MPP são os momentos-L amostrais, definidos pelas expressões 1 a 4 . Esses momentos-L de grau $r(r \leq 4)$ representam casos especiais de relevância para a inferência estatística. Os valores $b_{r}$, são os MPP amostrais.

$$
\begin{aligned}
& \mathrm{I}_{1}=\mathrm{b}_{0} \\
& \mathrm{I}_{2}=2 \mathrm{~b}_{1}-\mathrm{b}_{0} \\
& \mathrm{I}_{3}=6 \mathrm{~b}_{2}-6 \mathrm{~b}_{1}+\mathrm{b}_{0} \\
& \mathrm{I}_{4}=20 \mathrm{~b}_{3}-30 \mathrm{~b}_{2}+12 \mathrm{~b}_{1}-\mathrm{b}_{0}
\end{aligned}
$$

Em termos de medidas de forma das distribuições, torna-se mais conveniente que os momentos-L sejam expressos em quantidades adimensionais. Estas são representadas pelos quocientes de momentos- $\mathrm{L}$ amostrais, dados por

$$
t_{r}=\frac{I_{r}}{I_{2}} ; \text { para } r \geq 3
$$

Desta forma, $t_{3}$ e $t_{4}$ são, respectivamente, as medidas de assimetria e curtose, independentes da escala da distribuição de probabilidade. Pode-se definir também, em termos de momentos-L, uma medida análoga ao coeficiente de variação da seguinte forma:

$$
\mathrm{t}=\frac{\mathrm{I}_{2}}{\mathrm{I}_{1}}
$$

Todo estudo regional envolve análises subjetivas, principalmente nos estágios de identificação de regiões homogêneas e na escolha da distribuição de probabilidades teóricas e estimativa de seus parâmetros. Hosking e Wallis (1997) apresentaram três estatísticas, às quais denominaram estatísticas-L, como auxiliares nessas análises, sendo elas: medida de discordância (Di); medida de heterogeneidade $(\mathrm{H})$; e medida de aderência $(\mathrm{Z})$, as quais serão revistos nos itens subseqüentes.

\section{IDENTIFICAÇÃO DE REGIÕES HOMOGÊNEAS}

Uma região é considerada homogênea quando existem evidências suficientes de que as diferentes amostras do grupo possuem a mesma distribuição de freqüência à exceção de um fator de escala local.

De acordo com Hosking e Wallis (1997), a identificação destas regiões deve ser feita em duas etapas consecutivas: a primeira, consistindo de uma delimitação preliminar baseada unicamente nas características locais, e a segunda consistindo de um teste estatístico construído com base somente nas estatísticas locais, cujo objetivo é verificar os resultados preliminares obtidos.

\section{IDENTIFICAÇÃO PRELIMINAR DE REGIÕES HOMOGÊNEAS: ANÁLISE DE AGRUPAMENTO}

A análise de agrupamento tem por finalidade reunir, segundo um critério de classificação, os indivíduos (ou objetos, locais, populações, amostras, etc.) em vários grupos, de tal forma que exista homogeneidade dentro do grupo e heterogeneidade entre grupos (Cruz, 1997). Esta análise envolve basicamente duas etapas. A primeira diz respeito à estimação de uma medida de similaridade (ou dissimilaridade) entre os indivíduos ou populações a serem agrupados, e a segunda a adoção de um método de agrupamento para formação dos conglomerados (Cruz, 1997). O resultado depende da medida de dissimilaridade e do método de agrupamento escolhido.

O princípio das medidas de distância ou medidas de dissimilaridade considera que, se dois indivíduos são similares, eles estão próximos um do outro, ou seja, eles são comuns em relação ao conjunto de variáveis e viceversa (Liberato, 1995).

\section{MEDIDA DE DISCORDÂNCIA (DI)}

Em um grupo de amostras, a medida de discordância (Di) tem por objetivo identificar aquelas que apresentam características estatísticas muito discrepantes das grupais. Esta medida é expressa como uma estatística única, envolvendo as estimativas dos quocientes de momentos- $\mathrm{L}$ amostrais, sendo eles: coeficiente de variação- $\mathrm{L}(\mathrm{t})$, assimetria-L ( $\left.t_{3}\right)$ e curtose- $\mathrm{L}\left(\mathrm{t}_{4}\right)$. Em um espaço tridimensional de variação desses quocientes de momentos-L, a idéia é assinalar as amostras cujos valores $\left\{\mathrm{t}_{\mathrm{t}} \mathrm{t}_{3}, \mathrm{t}_{4}\right\}$ representados por um ponto no espaço, afastam-se demasiadamente do núcleo de concentração das amostras do grupo.

Algebricamente, considera-se que $u_{i}$ representa um vetor $(3 \mathrm{x} 1)$ contendo esses quocientes de momentos-L, dado pela expressão 7 , em que T indica uma matriz transposta.

$$
u_{i}=\left(\begin{array}{lll}
t^{i} & t_{3}^{i} & t_{4}^{i}
\end{array}\right)^{\top}
$$

Sendo $\overline{\mathrm{u}}$ um vetor $(3 \mathrm{x} 1)$ contendo a média grupal ou regional dos quocientes de momentos-L, tomada como a média aritmética simples de $\mathrm{u}_{\mathrm{i}}$ para todos os postos estudados, ele pode ser expresso por

$$
\overline{\mathrm{u}}=\frac{\sum_{\mathrm{i}=1}^{\mathrm{N}} \mathrm{u}_{\mathrm{i}}}{\mathrm{N}}=\left(\begin{array}{lll}
t^{\mathrm{R}} & \mathrm{t}_{3}^{\mathrm{R}} & \mathrm{t}_{4}^{\mathrm{R}}
\end{array}\right)^{\top}
$$

em que $\mathrm{N}$ representa o número de postos de observação do grupo ou região R em questão.

Dada a matriz de covariância amostral S, definida por 


$$
\mathrm{S}=(\mathrm{N}-1)^{-1} \sum_{\mathrm{i}=1}^{\mathrm{N}}\left(\mathrm{u}_{\mathrm{i}}-\overline{\mathrm{u}}\right)\left(\mathrm{u}_{\mathrm{i}}-\overline{\mathrm{u}}\right)^{\top}
$$

A medida de discordância Di, para o local i, pode ser definida pela equação

$$
D i=\frac{N}{3(N-1)}\left(u_{i}-\bar{u}\right)^{\top} S^{-1}\left(u_{i}-\bar{u}\right)
$$

Os valores limites de Di, utilizados como critério para decidir se a amostra é discordante das características grupais, são apresentados na Tabela 1.

De acordo com Hosking e Wallis (1997), para grupos ou regiões com um número muito reduzido de postos de observação, a estatística Di não é informativa. Por exemplo, para $\mathrm{N} \leq 3$ a matriz de covariância $\mathrm{S}$ é singular e o valor de Di não pode ser calculado. Para $\mathrm{N}=4$, Di $=1$, e para $\mathrm{N}=5$ ou $\mathrm{N}=6$ os valores de Di, como indicados no Quadro 1, são bastante próximos do limite algébrico da estatística, definido por $\mathrm{Di} \leq(\mathrm{N}-1) / 3$. Em conseqüência, os autores sugerem o uso da medida de discordância Di somente para $\mathrm{N} \geq 7$.

Tabela 1 - Valores críticos da medida de discordância - Di

\begin{tabular}{cc}
\hline $\begin{array}{c}\text { Número de postos na } \\
\text { região }(\mathrm{N})\end{array}$ & $\mathrm{Di}_{\text {crit }}$ \\
\hline 5 & 1,333 \\
6 & 1,648 \\
7 & 1,917 \\
8 & 2,140 \\
9 & 2,329 \\
10 & 2,491 \\
11 & 2,632 \\
12 & 2,757 \\
13 & 2,869 \\
14 & 2,971 \\
$\geq 15$ & 3 \\
\hline
\end{tabular}

Fonte: HOSKING e WALLIS (1997).

\section{MEDIDA DE HETEROGENEIDADE REGIONAL (H)}

Em uma região homogênea, todos os indivíduos possuem os mesmos quocientes de momentos-L populacionais. Entretanto, suas estimativas, ou seja, os quocientes de momentos-L calculados a partir das amostras, serão diferentes devido às flutuações amostrais (Hosking e Wallis, 1997).
Para tornar possível o cálculo das estatísticas simuladas para a região homogênea, é necessário especificar uma função de distribuição de probabilidades para a população da qual serão extraídas as amostras. Hosking e Wallis (1997) aconselham o emprego da distribuição Kappa de quatro parâmetros, baseando-se na premissa de que não se deve assumir, a priori, comprometimento com distribuições de dois ou três parâmetros. Os quocientes momentosL da distribuição Kappa populacional devem reproduzir as médias grupais dos quocientes CV-L ( $\mathrm{t}$ ), assimetria-L $\left(\mathrm{t}_{3}\right)$ e curtose $\left(\mathrm{t}_{4}\right)$, calculados para os dados observados.

A equação da distribuição Kappa é representada por

$$
\mathrm{F}(\mathrm{x})=\left\{1-\mathrm{h}\left[1-\frac{v(\mathrm{x}-\xi)}{\alpha}\right]^{\frac{1}{v}}\right\}^{\frac{1}{h}}
$$

A medida de heterogeneidade $\mathrm{H}$ compara a dispersão observada com a simulada, ou seja:

$$
H=\frac{V-\mu_{\operatorname{SIM}}}{\sigma_{\mathrm{SIM}}}
$$

em que $\sigma_{\text {SIM }}$ é o desvio padrão entre os $\mathrm{N}_{\text {sim }}$ valores da medida de dispersão $V_{\mathfrak{j}}$, ou seja,

$$
\sigma_{S I M}=\sqrt{\frac{\sum_{j=1}^{N_{S I M}}\left(v_{j}-\mu_{S I M}\right)^{2}}{N_{S I M}}}
$$

De acordo com os autores citados anteriormente, deve-se adotar o critério de classificação em que se $\mathrm{H}<$ 1,0, a região deve ser considerada homogênea; $1,0 \leq \mathrm{H} \leq$ 2,0, a região é possivelmente heterogênea; e se $H \geq 2,0$, a região é definitivamente heterogênea.

\section{TESTE PARAMÉTRICO DE ADERÊNCIA}

Em geral, a seleção da melhor distribuição de probabilidade baseia-se na qualidade e consistência de seu ajuste aos dados disponíveis. Para tal, existem diversos testes de aderência de uma distribuição aos dados amostrais, passíveis de serem adaptados ao contexto da análise regional de freqüência. Hosking e Wallis (1997) propuse- 
ram, em seus trabalhos, um teste de aderência denominado medida de aderência Z, para verificar se a distribuição candidata fornece um bom ajuste aos dados regionais, isto é, se os momentos- $\mathrm{L}$ regionais são coerentes como os da distribuição candidata.

Em termos gerais, essa medida de aderência fornece um valor para $Z$, que é função da relação entre a curtose-L da distribuição ajustada e a média regional da curtose-L amostral e do desvio padrão da curtose-L simulada, calculado para cada distribuição candidata, como mostra a equação 14. Aceita-se a distribuição candidata como adequada se $|Z| \leq 1,64$. Caso nenhuma das distribuições de três parâmetros atenda ao critério deve-se selecionar a distribuição Kappa de quatro parâmetros ou Wakeby de cinco parâmetros, por serem mais robustas.

$$
Z^{\mathrm{DIST}}=\frac{\tau_{4}^{\mathrm{DIST}}-\mathrm{t}_{4}^{\mathrm{R}}+\mathrm{B}_{4}}{\sigma_{4}}
$$

em que $\tau_{4}^{\text {DIST }}$ é a curtose-L da distribuição ajustada; DIST são as distribuições candidatas; $\mathrm{t}_{4}^{\mathrm{m}}$ são as médias regionais da curtose-L da m-ésima região simulada; $\mathrm{B}_{4}$ é o viés de $\mathrm{t}_{4}^{\mathrm{R}}$; e $\sigma_{4}$ é o desvio padrão de $\mathrm{t}_{4}^{\mathrm{R}}$.

Segundo os autores a estatística Z é especificada sob a forma de um teste de significância e possui distribuição que se aproxima da Normal padrão. Assim, o critério $|\mathrm{Z}| \leq 1,64$ corresponde à aceitação da hipótese de que os dados provêm da distribuição candidata, com um nível de confiança de $90 \%$

\section{CARACTERIZAÇÃO DA BACIA DO RIO DOCE}

A bacia do rio Doce situa-se na região sudeste, na faixa tropical do hemisfério sul, entre os paralelos 17॰30' e $21^{\circ}$ de latitude sul e os meridianos de $40^{\circ} 30^{\prime}$ e $44^{\circ}$ de longitude Oeste.

O rio Doce, cujas nascentes encontram-se na serra da Mantiqueira e no complexo do Espinhaço, em Minas Gerais, percorre $853 \mathrm{~km}$ até atingir o oceano Atlântico junto a cidade de Regência, no Estado do Espírito Santo. Sua área de drenagem abrange $83.431 \mathrm{~km}^{2}$, dos quais 86,1 $\%$ pertencem ao território mineiro e $13,9 \%$, ao capixaba.

A bacia do rio Doce apresenta uma ampla variação climática devida a elementos topográficos, que acentuam características variadas às massas de ar. No decorrer do ano, o comportamento do clima é bastante afetado pelo confronto entre a massa Polar e as massas continentais Tropical e Equatorial (Euclydes et al., 1996).

A distribuição das precipitações na bacia é heterogênea e, segundo Euclydes et al. (1996), ocorre maior concentração na área pertencente ao Estado do Espírito Santo, devido aos efeitos orográficos. Precipitações mais escassas ocorrem na área que se estende de Colatina (ES) até próximo a Governador Valadares (MG). A precipitação total anual varia de 900 a $1900 \mathrm{~mm}$ (período de 1948 a 1980).

\section{SELEÇÃO DAS ESTAÇÕES PLUVIOGRÁFICAS E ELABORAÇÃO DAS SÉRIES ANUAIS DE INTENSIDADES MÁXIMAS MÉDIAS DE PRECIPITAÇÃO}

Foram selecionadas 31 estações pluviográficas localizadas na bacia do rio Doce, pertencentes às redes hidrometeorológicas da ANA (Agência Nacional de Águas), do INMET (Instituto Nacional de Meteorologia) e da CEMIG (Companhia Energética de Minas Gerais).

Para a análise de freqüência das chuvas intensas, as séries de dados utilizadas foram do tipo anual, formadas pelos maiores valores observados em cada ano, desprezando-se os demais valores, ainda que superiores aos de outros anos. Desta forma, após a seleção dos pluviogramas com as chuvas mais intensas de cada ano da série histórica, foi realizada a contagem destes objetivando elaborar as séries anuais para as precipitações máximas com durações de $10,20,30,40,50,60,120,180,240,360,720$ e 1440 minutos, para cada uma das 31 estações selecionadas. Dividindo-se as alturas máximas precipitadas pela duração correspondente, obteve-se a intensidade máxima média de precipitação.

\section{APLICAÇÃO DA METODOLOGIA DE REGIONALIZAÇÃO BASEADA NOS MOMENTOS-L}

As estações pluviográficas foram agrupadas em cinco regiões utilizando-se os métodos aglomerativos hierárquicos de Ward e de K-médias ("K-means clustering"), tendo a distância Euclidiana ao quadrado e a distância Euclidiana simples como medidas de similaridade, respectivamente. $\mathrm{Na}$ seqüência, aplicou-se os critérios das análises estatísticas de medida de discordância (Di) e heterogeneidade $(\mathrm{H})$ às estações intragrupo verificando-se que estas foram não-discordantes e localizadas em uma região homogênea. Na Figura 1 é apresentado um mapa com a distribuição geográfica das 31 estações utilizadas no estudo e as respectivas regiões homogêneas obtidas e na Tabela 2 a identificação e detalhamento de todas as estações pluviográficas.

Ressalta-se que, neste trabalho, optou-se por denominar de região homogênea ao conjunto de estações homogêneas, visto que não houve delimitação de uma região propriamente dita. Assim, ao conjunto de postos com comportamento hidrológico semelhante, em uma 
determinada área, denominou-se como "região homogênea".

Entre as possíveis distribuições consideradas aceitáveis segundo o teste de aderência Z, a distribuição Generalizada Logística de três parâmetros foi a que apresentou melhor ajuste para a quase totalidade das durações estudadas, em todas as regiões homogêneas, à exceção de algumas durações em que a distribuição Wakeby de cinco parâmetros foi adotada, pois as demais distribuições não atenderam ao critério de $|Z| \leq 1,64$ e, por recomendação de Hosking e Wallis (1997), deve-se adotar uma distribuição de quatro ou cinco parâmetros por serem mais robustas.

As funções densidade de probabilidade, $\mathrm{f}(\mathrm{x})$, as funções de distribuição acumulada, $\mathrm{F}(\mathrm{x})$, as funções quantis, $x(F)$, os parâmetros da distribuição Generalizada Logística de três parâmetros $(\alpha=$ parâmetro de escala, $v=$ parâmetro de forma e $\xi=$ parâmetro de posição) e de Wakeby de cinco parâmetros ( $\alpha=$ parâmetro de escala, $v$, $\beta, \gamma=$ parâmetro de forma e $\xi=$ parâmetro de posição) são representados a seguir.

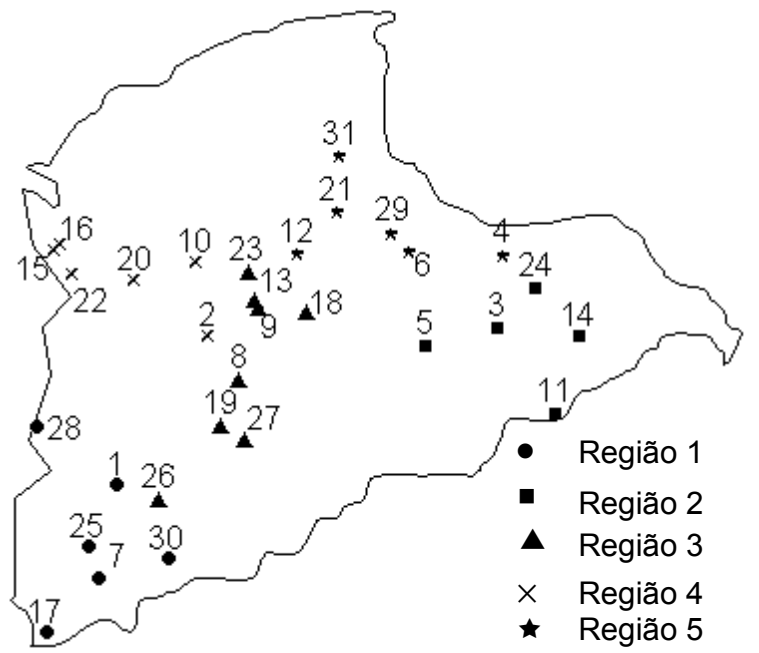

Figura 1 - Distribuição geográfica das estações pluviográficas na bacia do rio Doce e as respectivas regiões homogêneas obtidas.

a) Distribuição Generalizada Logística

$$
f(x)=\frac{e^{-(1-v) y}}{\alpha\left(1+e^{-y}\right)^{2}}
$$

em que

$$
\begin{aligned}
& y=\frac{\ln \left[1-\frac{v(x-\xi)}{\alpha}\right]}{v} \text { para } v \neq 0 \text { ou } \\
& y=\frac{x-\xi}{\alpha}, \text { para } v=0 \\
& F(x)=\frac{1}{\left(1+e^{-y}\right)}
\end{aligned}
$$

em que

$$
\begin{aligned}
& x(F)=\xi+\frac{\alpha}{v}\left[1-\left(\frac{1-F}{F}\right)^{v}\right], \operatorname{para} \nu \neq 0 \\
& \text { ou } x(F)=\xi-\alpha \ln \left[\frac{(1-F)}{F}\right], \operatorname{para} \nu=0
\end{aligned}
$$

- $\quad$ parâmetro de posição:

$$
\xi=\lambda_{1}-\alpha\left[\frac{1}{v}-\frac{\pi}{\operatorname{sen}(v \pi)}\right]
$$

- parâmetro de escala:

$$
\alpha=\frac{\lambda_{2} \operatorname{sen}(v \pi)}{v \pi}
$$

- parâmetro de forma:

$$
v=-\tau_{3}
$$

b) Distribuição Wakeby

Domínio de x:

$$
\begin{aligned}
& \xi \leq x<\infty \text { se } \gamma \geq 0 \text { e } \beta>0 \\
& \xi \leq x<\xi+\alpha / v-\beta / \gamma \text { se } \gamma<0 \text { ou } \gamma=0
\end{aligned}
$$


$f(x)$ e $F(x)$ não tem forma analítica explícita.

$$
x(F)=\xi+\frac{\alpha}{v}\left\{1-(1-F)^{v}\right) \frac{\beta}{\gamma}\left\{1-(1-F)^{-\gamma}\right\}
$$

Se $\xi$ for desconhecido, os parâmetros das distribuições, em termos de momentos-L, são dados pelas expressões apresentadas por HOSKING e WALLIS (1997).

Assim, v e - $\gamma$ são as raízes da equação quadrática

$$
\begin{aligned}
\left(\mathrm{N}_{2} \mathrm{C}_{3}-\mathrm{N}_{3} \mathrm{C}_{2}\right) \mathrm{z}^{2} & +\left(\mathrm{N}_{1} \mathrm{C}_{3}-\mathrm{N}_{3} \mathrm{C}_{1}\right) \mathrm{z} \\
+ & \left(\mathrm{N}_{1} \mathrm{C}_{2}-\mathrm{N}_{2} \mathrm{C}_{1}\right)=0
\end{aligned}
$$

Sendo $v$ o maior valor das duas raízes e os outros parâmetros são dados por:

- $\quad$ parâmetro de posição:

$$
\xi=\frac{\lambda_{1}-\alpha}{(1+v)-\frac{\beta}{(1-\gamma)}}
$$

- parâmetro de escala:

$$
\alpha=(1+v)(2+v)(3+v) \frac{(1+\gamma) \lambda_{2}-(3-\gamma) \lambda_{3}}{4(v+\gamma)}
$$

- parâmetro de forma:

$$
\beta=-(1-\gamma)(2-\gamma)(3-\gamma) \frac{(1-v) \lambda_{2}-(3+v) \lambda_{3}}{4(v+\gamma)}
$$

Se $\xi$ for conhecido, assume-se que $\xi=0$ sem perda de generalidade e utiliza-se as expressões para o

\begin{tabular}{|c|c|c|c|c|c|c|}
\hline $\mathrm{N}^{\mathrm{o}}$ & Estação & Código & $\begin{array}{l}\text { Lat. } \\
\left({ }^{\circ}\right)\end{array}$ & $\begin{array}{l}\text { Long. } \\
\left({ }^{\circ}\right)\end{array}$ & $\begin{array}{l}\text { Alt. } \\
(\mathrm{m})\end{array}$ & Região \\
\hline 1 & Acaiaca & 02043009 & 20,35 & 43,13 & 423 & 1 \\
\hline 2 & Acesita & 01942009 & 19,53 & 42,63 & 240 & 4 \\
\hline 3 & Aimorés & 01941017 & 19,48 & 41,06 & 83 & 2 \\
\hline 4 & Alto Rio Novo & 01941012 & 19,08 & 41,03 & 600 & 5 \\
\hline 5 & Assarai & 01941006 & 19,58 & 41,45 & 172 & 2 \\
\hline 6 & Barra do Cuiete & 01941005 & 19,06 & 41,53 & 121 & 5 \\
\hline 7 & Braz Pires & 02043026 & 20,85 & 43,23 & 632 & 1 \\
\hline 8 & $\begin{array}{l}\text { Cachoeira dos } \\
\text { Óculos }\end{array}$ & 01942031 & 19,76 & 42,46 & 425 & 3 \\
\hline 9 & $\begin{array}{l}\text { Cachoeira Escu- } \\
\text { ra }\end{array}$ & 01942001 & 19,81 & 42,31 & 242 & 3 \\
\hline 10 & $\begin{array}{l}\text { Usina } \\
\text { Grande }\end{array}$ & 01942025 & 19,13 & 42,7 & 430 & 4 \\
\hline 11 & Caldeirão & 01940020 & 19,95 & 40,75 & 690 & 2 \\
\hline 12 & Caratinga & 01942021 & 19,08 & 42,15 & 610 & 5 \\
\hline 13 & Cenibra & 01942030 & 19,33 & 42,38 & 242 & 3 \\
\hline 14 & Colatina & 01940006 & 19,53 & 40,61 & 40 & 2 \\
\hline 15 & $\begin{array}{l}\text { Conceição do } \\
\text { Mato Dentro }\end{array}$ & 01943002 & 19,06 & 43,46 & 675 & 4 \\
\hline 16 & $\begin{array}{l}\text { Conceição do } \\
\text { Mato Dentro }\end{array}$ & 01943059 & 19,03 & 43,43 & 652 & 4 \\
\hline 17 & $\begin{array}{l}\text { Desterro do } \\
\text { Melo }\end{array}$ & 02143003 & 21,15 & 43,51 & 780 & 1 \\
\hline 18 & Dom Cavati & 01942008 & 19,4 & 42,1 & 355 & 3 \\
\hline 19 & $\begin{array}{l}\text { Fazenda } \\
\text { Marias }\end{array}$ & 02042033 & 20,01 & 42,56 & 364 & 3 \\
\hline 20 & Ferros & 01943003 & 19,23 & 43,03 & 470 & 4 \\
\hline 21 & $\begin{array}{l}\text { Governador } \\
\text { Valadares }\end{array}$ & 01841015 & 18,85 & 41,93 & 278 & 5 \\
\hline 22 & Morro do Pilar & 01943025 & 19,2 & 43,36 & 560 & 4 \\
\hline 23 & Naque Velho & 01942032 & 19,18 & 42,41 & 240 & 3 \\
\hline 24 & Pancas & 01940009 & 19,26 & 40,85 & 135 & 2 \\
\hline 25 & Piranga & 02043010 & 20,68 & 43,28 & 620 & 1 \\
\hline 26 & Ponte Nova & 02042018 & 20,41 & 42,9 & 350 & 3 \\
\hline 27 & Raul Soares & 02042008 & 20,1 & 42,43 & 305 & 3 \\
\hline 28 & $\begin{array}{l}\text { São Gonçalo do } \\
\text { Rio Acima }\end{array}$ & 02043063 & 20,03 & 43,56 & 740 & 1 \\
\hline 29 & Tumiritinga & 01841011 & 18,96 & 41,63 & 138 & 5 \\
\hline 30 & Viçosa & 02042024 & 20,75 & 42,85 & 652 & 1 \\
\hline 31 & Vila Matias & 01841001 & 18,55 & 41,91 & 174 & 5 \\
\hline
\end{tabular}
cálculo dos parâmetros da distribuição, em termos de momentos-L, apresentadas por Hosking e Wallis (1997).
Tabela 2 - Identificação das estações pluviográficas localizadas na bacia do rio Doce e as respectivas regiões homogêneas obtidas 
Tabela 3 - Quantis adimensionais regionais ( $\left.\mathrm{q}_{\mathrm{T}, \mathrm{d}}\right)$ para a região homogênea 1

\begin{tabular}{cccccccc}
\hline Duração da precipitação (min) & \multicolumn{7}{c}{ Período de retorno (anos) } \\
\cline { 2 - 7 } & 2 & 5 & 10 & 20 & 50 & 75 & 100 \\
\hline 10 & 0,986 & 1,246 & 1,405 & 1,558 & 1,760 & 1,856 & 1,916 \\
20 & 0,984 & 1,235 & 1,390 & 1,540 & 1,739 & 1,834 & 1,893 \\
30 & 0,990 & 1,238 & 1,389 & 1,532 & 1,720 & 1,808 & 1,863 \\
40 & 0,990 & 1,237 & 1,386 & 1,528 & 1,713 & 1,800 & 1,854 \\
50 & 0,977 & 1,234 & 1,397 & 1,558 & 1,776 & 1,883 & 1,949 \\
60 & 0,972 & 1,232 & 1,400 & 1,568 & 1,797 & 1,911 & 1,982 \\
120 & 0,951 & 1,234 & 1,431 & 1,637 & 1,936 & 2,090 & 2,189 \\
180 & 0,940 & 1,227 & 1,434 & 1,657 & 1,989 & 2,165 & 2,279 \\
240 & 0,935 & 1,227 & 1,441 & 1,673 & 2,024 & 2,211 & 2,333 \\
360 & $0,930 *$ & $1,199 *$ & $1,433 *$ & $1,698^{*}$ & $2,102^{*}$ & $2,315^{*}$ & $2,454^{*}$ \\
720 & $0,919 *$ & $1,201^{*}$ & $1,442^{*}$ & $1,711^{*}$ & $2,114 *$ & $2,325^{*}$ & $2,460^{*}$ \\
1440 & 0,937 & 1,229 & 1,442 & 1,672 & 2,017 & 2,200 & 2,319 \\
\hline
\end{tabular}

* Distribuição Wakeby de cinco parâmetros.

Tabela 4 - Quantis adimensionais regionais $\left(\mathrm{q}_{\mathrm{T}, \mathrm{d}}\right)$ para a região homogênea 2

\begin{tabular}{cccccccc}
\hline Duração da precipitação (min) & \multicolumn{9}{c}{ Período de retorno (anos) } \\
\cline { 2 - 7 } & 2 & 5 & 10 & 20 & 50 & 75 & 100 \\
\hline 10 & 0,976 & 1,236 & 1,401 & 1,564 & 1,785 & 1,893 & 1,960 \\
20 & $0,995^{*}$ & $1,180^{*}$ & $1,335^{*}$ & $1,506^{*}$ & $1,762^{*}$ & $1,895^{*}$ & $1,980^{*}$ \\
30 & $1,003^{*}$ & $1,184^{*}$ & $1,338^{*}$ & $1,514^{*}$ & $1,788^{*}$ & $1,936^{*}$ & $2,032^{*}$ \\
40 & $1,010^{*}$ & $1,196^{*}$ & $1,349^{*}$ & $1,525^{*}$ & $1,795^{*}$ & $1,940^{*}$ & $2,034^{*}$ \\
50 & $0,992^{*}$ & $1,226^{*}$ & $1,408^{*}$ & $1,600^{*}$ & $1,872^{*}$ & $2,006^{*}$ & $2,091^{*}$ \\
60 & 0,971 & 1,259 & 1,445 & 1,629 & 1,880 & 2,004 & 2,082 \\
120 & 0,937 & 1,262 & 1,492 & 1,736 & 2,095 & 2,283 & 2,405 \\
180 & 0,931 & 1,250 & 1,482 & 1,732 & 2,108 & 2,307 & 2,437 \\
240 & 0,928 & 1,248 & 1,483 & 1,737 & 2,123 & 2,329 & 2,463 \\
360 & 0,931 & 1,246 & 1,477 & 1,725 & 2,099 & 2,298 & 2,428 \\
720 & 0,934 & 1,248 & 1,475 & 1,718 & 2,081 & 2,273 & 2,397 \\
1440 & 0,960 & 1,262 & 1,463 & 1,667 & 1,954 & 2,098 & 2,189 \\
\hline
\end{tabular}

* Distribuição Wakeby de cinco parâmetros.

Tabela 5 - Quantis adimensionais regionais $\left(\mathrm{q}_{\mathrm{T}, \mathrm{d}}\right)$ para a região homogênea 3

\begin{tabular}{cccccccc}
\hline Duração da precipitação (min) & \multicolumn{7}{c}{ Período de retorno (anos) } \\
\cline { 2 - 7 } & 2 & 5 & 10 & 20 & 50 & 75 & 100 \\
\hline 10 & 0,982 & 1,263 & 1,438 & 1,606 & 1,830 & 1,937 & 2,003 \\
20 & 0,981 & 1,246 & 1,413 & 1,574 & 1,790 & 1,895 & 1,959 \\
30 & 0,982 & 1,239 & 1,399 & 1,553 & 1,760 & 1,859 & 1,920 \\
40 & 0,976 & 1,239 & 1,408 & 1,573 & 1,798 & 1,908 & 1,976 \\
50 & 0,979 & 1,244 & 1,412 & 1,574 & 1,792 & 1,898 & 1,964 \\
60 & 0,978 & 1,250 & 1,423 & 1,591 & 1,817 & 1,927 & 1,995 \\
120 & 0,971 & 1,253 & 1,434 & 1,614 & 1,860 & 1,981 & 2,057 \\
180 & 0,972 & 1,264 & 1,452 & 1,637 & 1,888 & 2,011 & 2,088 \\
240 & 0,969 & 1,267 & 1,460 & 1,650 & 1,912 & 2,040 & 2,121 \\
360 & 0,964 & 1,277 & 1,481 & 1,685 & 1,967 & 2,108 & 2,196 \\
720 & 0,972 & 1,269 & 1,460 & 1,648 & 1,903 & 2,029 & 2,107 \\
1440 & 0,973 & 1,267 & 1,456 & 1,640 & 1,892 & 2,014 & 2,091 \\
\hline
\end{tabular}

* Distribuição Wakeby de cinco parâmetros. 
Tabela 6 - Quantis adimensionais regionais $\left(\mathrm{q}_{\mathrm{T}, \mathrm{d}}\right)$ para a região homogênea 4

\begin{tabular}{cccccccc}
\hline Duração da precipitação (min) & \multicolumn{7}{c}{ Período de retorno (anos) } \\
\cline { 2 - 7 } & 2 & 5 & 10 & 20 & 50 & 75 & 100 \\
\hline 10 & 0,989 & 1,219 & 1,359 & 1,494 & 1,670 & 1,754 & 1,806 \\
20 & 0,962 & 1,245 & 1,435 & 1,627 & 1,897 & 2,033 & 2,119 \\
30 & 0,990 & 1,233 & 1,381 & 1,522 & 1,705 & 1,791 & 1,845 \\
40 & 0,989 & 1,231 & 1,379 & 1,519 & 1,703 & 1,790 & 1,843 \\
50 & 0,984 & 1,231 & 1,385 & 1,534 & 1,732 & 1,827 & 1,886 \\
60 & $0,989 *$ & $1,204^{*}$ & $1,375^{*}$ & $1,561 *$ & $1,832^{*}$ & $1,970^{*}$ & $2,058^{*}$ \\
120 & 0,956 & 1,236 & 1,428 & 1,625 & 1,907 & 2,051 & 2,142 \\
180 & 0,941 & 1,238 & 1,449 & 1,674 & 2,007 & 2,181 & 2,294 \\
240 & 0,945 & 1,239 & 1,447 & 1,667 & 1,989 & 2,157 & 2,265 \\
360 & 0,933 & 1,240 & 1,465 & 1,707 & 2,072 & 2,266 & 2,392 \\
720 & 0,926 & 1,228 & 1,454 & 1,703 & 2,085 & 2,291 & 2,427 \\
1440 & $0,929 *$ & $1,204 *$ & $1,432 *$ & $1,678^{*}$ & $2,023 *$ & $2,217 *$ & $2,332^{*}$ \\
\hline
\end{tabular}

* Distribuição Wakeby de cinco parâmetros.

Tabela 7 - Quantis adimensionais regionais $\left(\mathrm{q}_{\mathrm{T}, \mathrm{d}}\right)$ para a região homogênea 5

\begin{tabular}{cccccccc}
\hline Duração da precipitação (min) & \multicolumn{9}{c}{ Período de retorno (anos) } \\
\cline { 2 - 7 } & 2 & 5 & 10 & 20 & 50 & 75 & 100 \\
\hline 10 & 0,989 & 1,228 & 1,374 & 1,512 & 1,693 & 1,779 & 1,832 \\
20 & $0,985^{*}$ & $1,195^{*}$ & $1,358^{*}$ & $1,526^{*}$ & $1,754^{*}$ & $1,864^{*}$ & $1,933^{*}$ \\
30 & $0,970^{*}$ & $1,198^{*}$ & $1,376^{*}$ & $1,560^{*}$ & $1,811^{*}$ & $1,933^{*}$ & $2,009^{*}$ \\
40 & $0,967^{*}$ & $1,212^{*}$ & $1,404^{*}$ & $1,603^{*}$ & $1,877^{*}$ & $2,011^{*}$ & $2,093^{*}$ \\
50 & 0,964 & 1,244 & 1,429 & 1,617 & 1,879 & 2,011 & 2,094 \\
60 & 0,957 & 1,247 & 1,443 & 1,644 & 1,928 & 2,072 & 2,164 \\
120 & 0,951 & 1,262 & 1,474 & 1,693 & 2,006 & 2,166 & 2,268 \\
180 & 0,953 & 1,266 & 1,479 & 1,696 & 2,006 & 2,163 & 2,263 \\
240 & 0,951 & 1,264 & 1,478 & 1,699 & 2,015 & 2,177 & 2,280 \\
360 & 0,959 & 1,263 & 1,466 & 1,672 & 1,960 & 2,105 & 2,197 \\
720 & 0,945 & 1,254 & 1,470 & 1,695 & 2,023 & 2,193 & 2,301 \\
1440 & 0,950 & 1,257 & 1,468 & 1,687 & 2,003 & 2,165 & 2,268 \\
\hline
\end{tabular}

Distribuição Wakeby de cinco parâmetros.

Assim, $v$ e $-\gamma$ são as raízes da equação quadrática 22 , sendo $v$ o maior valor das duas raízes da equação, sendo os outros parâmetros dados por:

- parâmetro de escala:

$$
\alpha=(1+v)(2+v) \frac{\lambda_{1}-(2-\gamma) \lambda_{2}}{(v+\gamma)}
$$

- $\quad$ parâmetro de forma:

$$
\beta=-(1-\gamma)(2-\gamma) \frac{\lambda_{1}-(2+v) \lambda_{2}}{(v+\gamma)}
$$

\section{ESTIMATIVA DOS PARÂMETROS DA DISTRI- BUIÇÃO REGIONAL E DOS QUANTIS ADIMEN- SIONAIS}

Com os parâmetros das distribuições regionais Generalizada Logística e Wakeby, calculados a partir da série de dados adimensionais, para as cinco regiões homogêneas, foram estimados os quantis regionais adimensionais de freqüência ( $\mathrm{q}_{\mathrm{T}, \mathrm{d}}$ ), cujos valores são apresentados nas Tabelas 3 a 7 . Esses quantis foram calculados para os períodos de retorno de dois, cinco, 10, 20, 50, 75 e 100 anos e durações de precipitação de 10, 20, 30, 40, 50, 60, 120, 180, 240, 360, 720 e 1440 minutos, aplicando-se as referidas distribuições de probabilidade selecionadas, sendo seus valores utilizados nas equações regionais de intensidade, duração e freqüência para efetuar o cálculo de intensidade máxima média de precipitação, por meio da equação 
Tabela 8 - Equações de regressão da variável dependente $\bar{i}_{d}$ ("index-flood") em função da duração da precipitação, da altitude e da altura de precipitação média anual para as cinco regiões homogêneas

\begin{tabular}{|c|c|c|}
\hline Região & Duração (min.) & Equação de regressão \\
\hline \multirow{3}{*}{1} & $10 \leq \mathrm{d} \leq 1440$ & $\begin{array}{c}\overline{\mathrm{i}}_{\mathrm{d}}=78,188 \mathrm{~d}^{-0,518} \mathrm{~A}^{-0,031} \mathrm{P}_{\mathrm{i}}^{0,236} \\
\left(\mathrm{r}_{\hat{\mathrm{i}} \mathrm{i}}=0,98\right)\end{array}$ \\
\hline & $10 \leq \mathrm{d}<60$ & $\begin{array}{c}\overline{\mathrm{i}}_{\mathrm{d}}=67,863 \quad \mathrm{~d}^{-0,388} \quad \mathrm{~A}^{-0,049} \quad \mathrm{P}_{\mathrm{i}}^{0,221} \\
\left(\mathrm{r}_{\mathrm{i} \hat{\mathrm{i}}}=0,97\right)\end{array}$ \\
\hline & $60 \leq \mathrm{d} \leq 1440$ & $\begin{array}{c}\overline{\mathrm{i}}_{\mathrm{d}}=160,182 \quad \mathrm{~d}^{-0,763} \quad \mathrm{~A}^{-0,039} \quad \mathrm{P}_{\mathrm{i}}^{0,290} \\
\left(\mathrm{r}_{\hat{i} \hat{\mathrm{i}}}=0,99\right)\end{array}$ \\
\hline \multirow{3}{*}{2} & $10 \leq \mathrm{d} \leq 1440$ & $\begin{array}{c}\overline{\mathrm{i}}_{\mathrm{d}}=282.135,6 \quad \mathrm{~d}^{-0,527} \quad \mathrm{~A}^{0,017} \quad \mathrm{P}_{\mathrm{j}}^{-0,964} \\
\left(\mathrm{r}_{\mathrm{i} \hat{\mathrm{i}}}=0,98\right)\end{array}$ \\
\hline & $10 \leq \mathrm{d}<60$ & $\begin{array}{c}\overline{\mathrm{i}}_{\mathrm{d}}=264.775,2 \quad \mathrm{~d}^{-0,403} \quad \mathrm{~A}^{0,014} \quad \mathrm{P}_{\mathrm{i}}^{-1,00} \\
\left(\mathrm{r}_{\mathrm{i} \hat{\mathrm{i}}}=0,97\right)\end{array}$ \\
\hline & $60 \leq \mathrm{d} \leq 1440$ & $\begin{array}{c}\bar{i}_{d}=62.663,16 \quad d^{-0,777} \quad A^{0,045} \quad P_{i}^{-0,611} \\
\left(r_{i} \hat{i}=0,99\right)\end{array}$ \\
\hline \multirow{3}{*}{3} & $10 \leq \mathrm{d} \leq 1440$ & $\begin{array}{c}\overline{\mathrm{i}}_{\mathrm{d}}=598,342 \quad \mathrm{~d}^{-0,523} \mathrm{~A}^{0,044} \mathrm{P}_{\mathrm{i}}^{-0,105} \\
\left(\mathrm{r}_{\mathrm{i} \hat{\mathrm{i}}}=0,98\right)\end{array}$ \\
\hline & $10 \leq \mathrm{d}<60$ & $\begin{array}{c}\overline{\mathrm{i}}_{\mathrm{d}}=625,812 \quad \mathrm{~d}^{-0,408} \quad \mathrm{~A}^{0,037} \quad \mathrm{P}_{\mathrm{i}}^{-0,151} \\
\left(\mathrm{r}_{\mathrm{i} \hat{\mathrm{i}}}=0,95\right)\end{array}$ \\
\hline & $60 \leq \mathrm{d} \leq 1440$ & $\begin{array}{c}\overline{\mathrm{i}}_{\mathrm{d}}=71,396 \mathrm{~d}^{-0,750} \quad \mathrm{~A}^{0,103} \mathrm{P}_{\mathrm{i}}^{0,283} \\
\left(\mathrm{r}_{\mathrm{i} \hat{\mathrm{i}}}=0,99\right)\end{array}$ \\
\hline \multirow{3}{*}{4} & $10 \leq \mathrm{d} \leq 1440$ & $\begin{array}{c}\overline{\mathrm{i}}_{\mathrm{d}}=1.469,119 \quad \mathrm{~d}^{-0,521} \quad \mathrm{~A}^{-0,123} \quad \mathrm{P}_{\mathrm{i}}^{-0,086} \\
\left(\mathrm{r}_{\mathrm{i} \hat{\mathrm{i}}}=0,98\right)\end{array}$ \\
\hline & $10 \leq \mathrm{d}<60$ & $\begin{array}{c}\overline{\mathrm{i}}_{\mathrm{d}}=1.570,998 \quad \mathrm{~d}^{-0,403} \quad \mathrm{~A}^{-0,123} \quad \mathrm{P}_{\mathrm{i}}^{-0,141} \\
\left(\mathrm{r}_{\mathrm{i} \hat{\mathrm{i}}}=0,97\right)\end{array}$ \\
\hline & $60 \leq \mathrm{d} \leq 1440$ & $\begin{array}{c}\overline{\mathrm{i}}_{\mathrm{d}}=231,864 \quad \mathrm{~d}^{-0,748} \quad \mathrm{~A}^{-0,133} \quad \mathrm{P}_{\mathrm{i}}^{0,312} \\
\left(\mathrm{r}_{\mathrm{i} \hat{\mathrm{i}}}=0,99\right)\end{array}$ \\
\hline \multirow{3}{*}{5} & $10 \leq \mathrm{d} \leq 1440$ & $\begin{array}{c}\overline{\mathrm{i}}_{\mathrm{d}}=23,135 \mathrm{~d}^{-0,504} \mathrm{~A}^{-0,044} \mathrm{P}_{\mathrm{j}}^{0,424} \\
\left(\mathrm{r}_{\mathrm{i} \hat{\mathrm{i}}}=0,97\right)\end{array}$ \\
\hline & $10 \leq \mathrm{d}<60$ & $\begin{array}{c}\overline{\mathrm{i}}_{\mathrm{d}}=19,927 \quad \mathrm{~d}^{-0,371} \quad \mathrm{~A}^{-0,035} \quad \mathrm{P}_{\mathrm{i}}^{0,384} \\
\left(\mathbf{r}_{\mathrm{i} \hat{\mathrm{i}}}=0,94\right)\end{array}$ \\
\hline & $60 \leq \mathrm{d} \leq 1440$ & $\begin{array}{c}\overline{\mathrm{i}}_{\mathrm{d}}=7,987 \quad \mathrm{~d}^{-0,763} \quad \mathrm{~A}^{-0,106} \quad \mathrm{P}_{\mathrm{i}}^{0,792} \\
\left(\mathrm{r}_{\mathrm{i}} \hat{\mathrm{i}}=0,97\right)\end{array}$ \\
\hline
\end{tabular}

$$
\hat{i}_{T, d, j}=\beta_{0} d^{\beta_{1}} A_{j}^{\beta_{2}} P_{j}^{\beta_{3}} q_{T, d}
$$

em que $\hat{\mathrm{i}}_{\mathrm{T}, \mathrm{d}, \mathrm{j}}=$ estimador da intensidade máxima média de precipitação $\left(\mathrm{mm} \mathrm{h}^{-1}\right)$, de duração d (min), no local j dentro de uma região homogênea, associado ao período de retor- no T (anos); $A_{j}=$ altitude do local $j(m) ; P_{j}=$ precipitação média anual do local j em estudo; $\mathrm{q}_{\mathrm{T}, \mathrm{d}}=$ quantil adimensional regional de freqüência associado a uma duração $\mathrm{d}$ e a um período de retorno $T$, de validade regional; e $\beta_{0}, \beta_{1}, \beta_{2}$, $\beta_{3}, \beta_{4}=$ parâmetros estimados para cada região homogê nea.

Tabela 9 - Equações de regressão da variável dependente $\overline{\mathrm{i}}_{d}$ (“index-flood") em função da duração da precipitação e da altura de precipitação média anual para as cinco regiões homogêneas

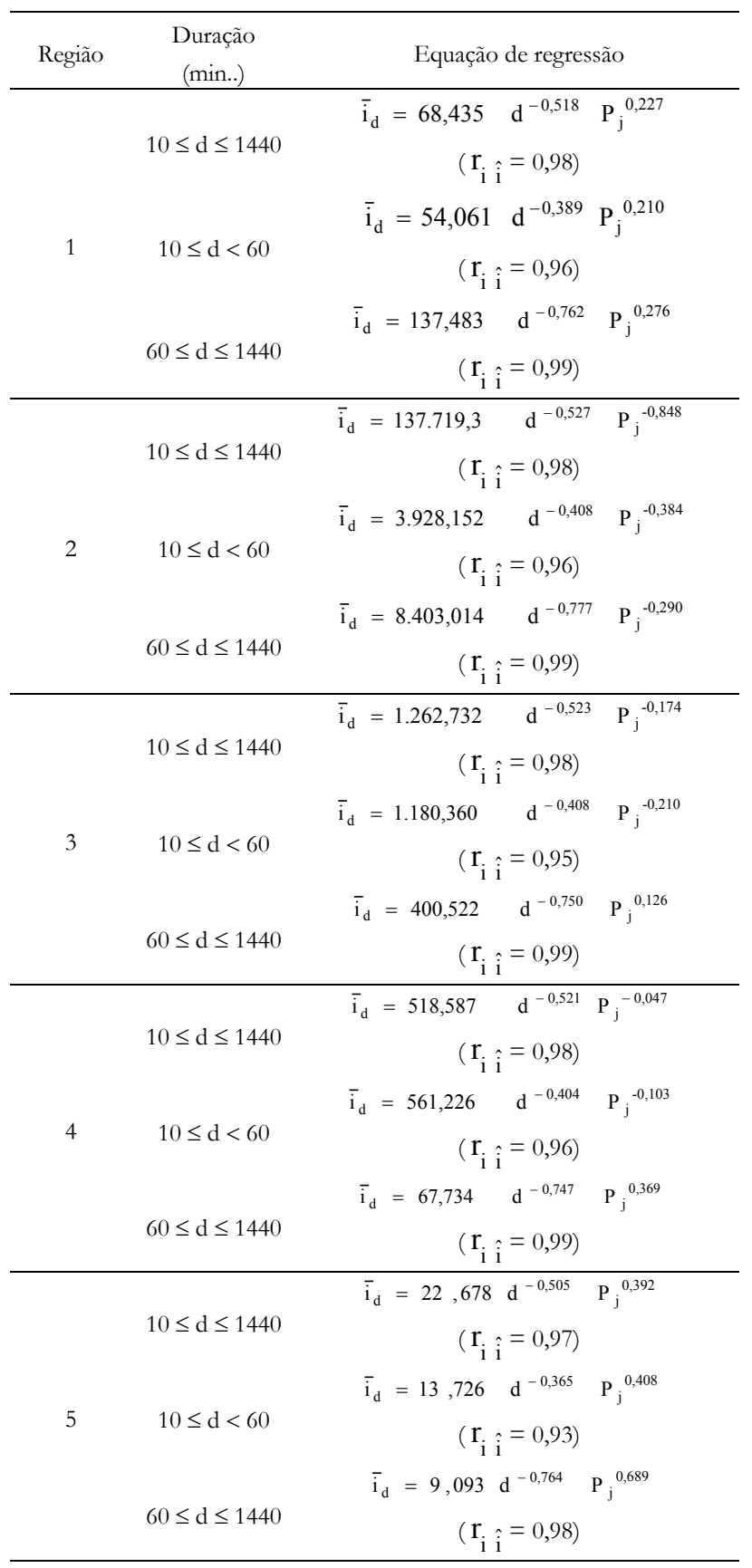




\section{EQUAÇÃO DE INTENSIDADE, DURAÇÃO E FREQÜÊNCIA REGIONAL}

Na Tabela 8 são apresentados os resultados da análise de regressão para as regiões homogêneas, tendo como variável dependente o fator "index-flood" ( $\left.\overline{\mathbf{i}}_{\mathrm{d}}\right)$ e como variáveis independentes a duração da precipitação (d), a altitude $\left(A_{j}\right)$ e a altura de precipitação média anual $\left(\mathrm{P}_{\mathrm{j}}\right)$. Na Tabela 9 são apresentadas outras equações em que a altitude foi excluída de todos os modelos de regressão por não proporcionar melhoria expressiva à regressão de $\mathrm{i}_{\mathrm{d}}$.

Tabela 10 - Equações regionais de intensidadeduração-freqüência para a bacia do rio Doce em função da duração da precipitação, da altura de precipitação média anual e do quantil adimensional regional para as cinco regiões homogêneas

\begin{tabular}{|c|c|c|}
\hline $\begin{array}{l}\text { Regi- } \\
\text { ão }\end{array}$ & $\begin{array}{l}\text { Duração } \\
\text { (min.) }\end{array}$ & $\begin{array}{l}\text { Equação regional do tipo intensida- } \\
\text { de-duração-freqüência }\end{array}$ \\
\hline 1 & $\begin{array}{l}10 \leq \mathrm{d} \leq \\
1440 \\
10 \leq \mathrm{d}< \\
60 \\
60 \leq \mathrm{d} \leq \\
1440\end{array}$ & $\begin{array}{l}\hat{\mathrm{i}}_{\mathrm{T}, \mathrm{d}, \mathrm{j}}=68,435 \mathrm{~d}^{-0,518} \mathrm{P}_{\mathrm{j}}^{0,227} \mathrm{q}_{\mathrm{T}, \mathrm{d}} \\
\hat{\mathrm{i}}_{\mathrm{T}, \mathrm{d}, \mathrm{j}}=54,061 \mathrm{~d}^{-0,389} \mathrm{P}_{\mathrm{j}}^{0,210} \mathrm{q}_{\mathrm{T}, \mathrm{d}} \\
\hat{\mathrm{i}}_{\mathrm{T}, \mathrm{d}, \mathrm{j}}=137,483 \mathrm{~d}^{-0,762} \mathrm{P}_{\mathrm{j}}^{0,276} \mathrm{q}_{\mathrm{T}, \mathrm{d}}\end{array}$ \\
\hline 2 & $\begin{array}{l}10 \leq \mathrm{d} \leq \\
1440 \\
10 \leq \mathrm{d}< \\
60 \\
60 \leq \mathrm{d} \leq \\
1440\end{array}$ & $\begin{array}{l}\hat{\mathrm{i}}_{\mathrm{T}, \mathrm{d}, \mathrm{j}}=137.719,3 \mathrm{~d}^{-0,527} \mathrm{P}_{\mathrm{j}}^{-0,848} \mathrm{q}_{\mathrm{T}, \mathrm{d}} \\
\hat{\mathrm{i}}_{\mathrm{T}, \mathrm{d}, \mathrm{j}}=3.928,152 \mathrm{~d}^{-0,408} \mathrm{P}_{\mathrm{j}}^{-0,384} \mathrm{q}_{\mathrm{T}, \mathrm{d}} \\
\hat{\mathrm{i}}_{\mathrm{T}, \mathrm{d}, \mathrm{j}}=8.403,014 \mathrm{~d}^{-0,777} \mathrm{P}_{\mathrm{j}}^{-0,290} \mathrm{q}_{\mathrm{T}, \mathrm{d}}\end{array}$ \\
\hline 3 & $\begin{array}{l}10 \leq \mathrm{d} \leq \\
1440 \\
10 \leq \mathrm{d}< \\
60 \\
60 \leq \mathrm{d} \leq \\
1440\end{array}$ & $\begin{array}{l}\hat{\mathrm{i}}_{\mathrm{T}, \mathrm{d}, \mathrm{j}}=1.262,732 \mathrm{~d}^{-0,523} \mathrm{P}_{\mathrm{j}}^{-0,174} \mathrm{q}_{\mathrm{T}, \mathrm{d}} \\
\hat{\mathrm{i}}_{\mathrm{T}, \mathrm{d}, \mathrm{j}}=1.180,360 \mathrm{~d}^{-0,408} \mathrm{P}_{\mathrm{j}}^{-0,210} \mathrm{q}_{\mathrm{T}, \mathrm{d}} \\
\hat{\mathrm{i}}_{\mathrm{T}, \mathrm{d}, \mathrm{j}}=400,522 \mathrm{~d}^{-0,750} \mathrm{P}_{\mathrm{j}}^{0,126} \mathrm{q}_{\mathrm{T}, \mathrm{d}}\end{array}$ \\
\hline 4 & $\begin{array}{l}10 \leq \mathrm{d} \leq \\
1440 \\
10 \leq \mathrm{d}< \\
60 \\
60 \leq \mathrm{d} \leq \\
1440\end{array}$ & $\begin{array}{l}\hat{\mathrm{i}}_{\mathrm{T}, \mathrm{d}, \mathrm{j}}=518,587 \mathrm{~d}^{-0,521} \mathrm{P}_{\mathrm{j}}^{-0,047} \mathrm{q}_{\mathrm{T}, \mathrm{d}} \\
\hat{\mathrm{i}}_{\mathrm{T}, \mathrm{d}, \mathrm{j}}=561,226 \mathrm{~d}^{-0,404} \mathrm{P}_{\mathrm{j}}^{-0,103} \mathrm{q}_{\mathrm{T}, \mathrm{d}} \\
\hat{\mathrm{i}}_{\mathrm{T}, \mathrm{d}, \mathrm{j}}=67,734 \mathrm{~d}^{-0,747} \mathrm{P}_{\mathrm{j}}^{0,369} \mathrm{q}_{\mathrm{T}, \mathrm{d}}\end{array}$ \\
\hline 5 & $\begin{array}{l}10 \leq \mathrm{d} \leq \\
1440 \\
10 \leq \mathrm{d}< \\
60 \\
60 \leq \mathrm{d} \leq \\
1440\end{array}$ & $\begin{array}{l}\hat{\mathrm{i}}_{\mathrm{T}, \mathrm{d}, \mathrm{j}}=22,678 \mathrm{~d}^{-0,505} \mathrm{P}_{\mathrm{j}}^{0,392} \mathrm{q}_{\mathrm{T}, \mathrm{d}} \\
\hat{\mathrm{i}}_{\mathrm{T}, \mathrm{d}, \mathrm{j}}=13,726 \mathrm{~d}^{-0,365} \mathrm{P}_{\mathrm{j}}^{0,408} \mathrm{q}_{\mathrm{T}, \mathrm{d}} \\
\hat{\mathrm{i}}_{\mathrm{T}, \mathrm{d}, \mathrm{j}}=9,093 \mathrm{~d}^{-0,764} \mathrm{P}_{\mathrm{j}}^{0,689} \mathrm{q}_{\mathrm{T}, \mathrm{d}}\end{array}$ \\
\hline
\end{tabular}

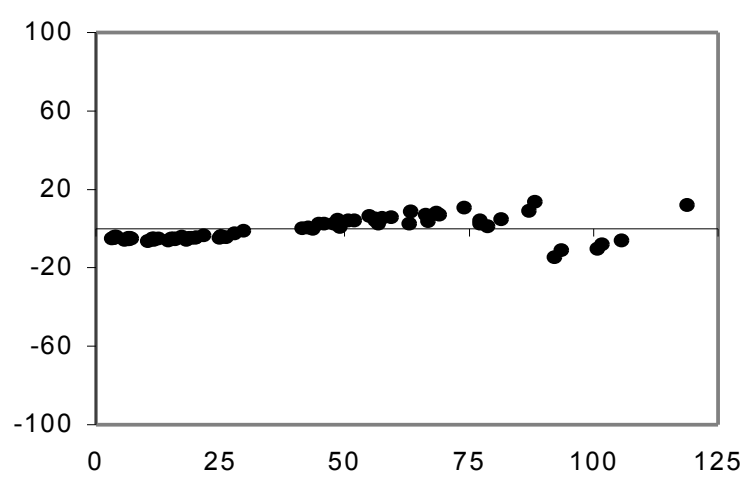

Valores residuais versus Observados - duração de 10 a $1440 \mathrm{~min}$

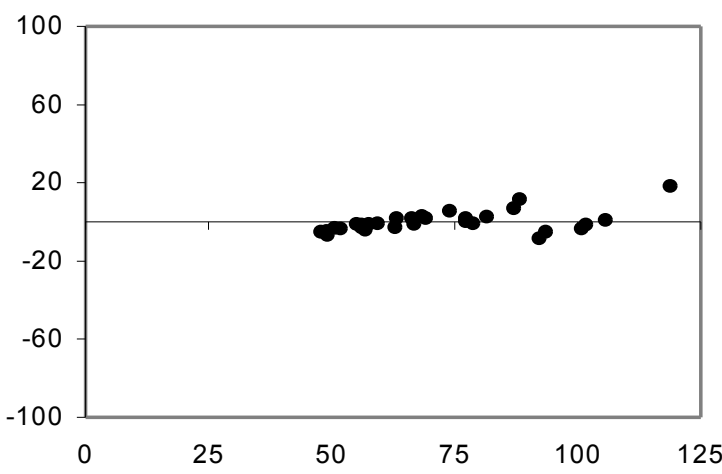

Valores residuais versus Observados - duração de 10 a 60 $\min$

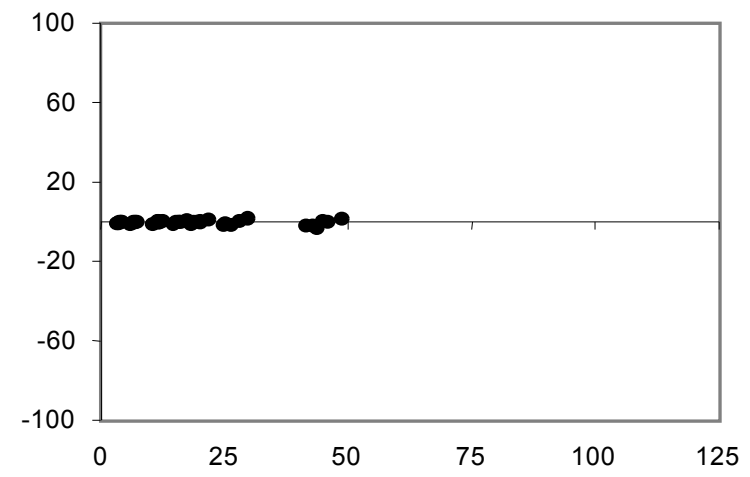

Valores residuais versus Observados - duração de 60 a $1440 \mathrm{~min}$

Figura 2 - Análise de resíduos para $\overline{\mathrm{i}}_{d}$ em função da duração e da precipitação média anual para a região homogênea 1.

Verifica-se na Figura 2, referente à região homogênea 1, que a equação regional que engloba todas as durações analisadas (10 a 1440 minutos) tende a subestimar os 
resultados para durações maiores ou iguais a 60 minutos, enquanto para as durações menores que 60 minutos ela tende a superestimar. Este comportamento também foi verificado para as demais regiões homogêneas.

Tendo em vista este fato, apesar da equação de regressão obtida para durações de precipitação de 10 a 1440 minutos nas diferentes regiões homogêneas apresentar alto coeficiente de correlação, optou-se por individualizá-la em duas equações, uma de 10 a 60 minutos e outra de 60 a 1440 minutos, devido ao fato das distribuições de resíduos destas últimas terem apresentado comportamento melhor.

A seleção da melhor equação foi feita com base no coeficiente de correlação $\left(r_{i} \hat{i}\right)$ entre os valores observados de intensidade máxima média de precipitação (i) e os valores estimados pela equação de regressão $(\hat{i})$ e na análise de resíduos.

Combinando as equações de regressão de $\overline{\mathrm{i}}_{\mathrm{d}}$, apresentadas na Tabela 9, com os quantis regionais adimensionais ( $\mathrm{q}_{\mathrm{T}, \mathrm{d}}$ ), dos Quadros 3 a 7, foram obtidas as equações de intensidade, duração e freqüência regionais para a Bacia do Rio Doce (Quadro 10).

Observa-se, na Tabela 10, uma variação considerável nos valores das estimativas de $\beta_{0}$ e $\beta_{3}$ das equações regionais de intensidade, duração e freqüência entre as regiões, e também para o parâmetro $\beta_{0}$ entre as equações correspondentes aos limites de suas durações dentro de uma mesma região homogênea, o que evidencia a necessidade de se obter equações específicas para cada região homogênea e de individualizá-las de acordo com as durações da precipitação.

\section{CONCLUSÕES}

A análise dos resultados obtidos para a bacia do rio Doce permitiu as seguintes conclusões:

- a distribuição probabilística de três parâmetros que apresentou melhor aderência aos dados de intensidade máxima média de precipitação, foi a Generalizada Logísti$\mathrm{ca}$;

- a distribuição de resíduos para as equações de intensidade, duração e freqüência, individualizadas para as durações de 10 a 60 minutos e de 60 a 1440 minutos, apresentou comportamento melhor do que a equação obtida para o período de 10 a 1440 minutos;

- o uso da altitude não proporcionou melhoria expressiva nos modelos de regressão do fator "indexflood" ' $\overline{\mathrm{i}}_{\mathrm{d}}$;

- os parâmetros das equações regionais de intensidade, duração e freqüência, ajustadas para as cinco regiões homogêneas, apresentaram variação acentuada entre as regiões, o que evidencia a importância de se obter equações específicas para cada região homogênea; e
- o método de regionalização hidrológica aplicado neste trabalho, permite determinar equações de intensidade, duração e freqüência para qualquer local dentro da região homogênea, bastando para isso conhecer a precipitação média anual.

\section{REFERÊNCIAS}

CRUZ, C.D. Modelos biométricos aplicados ao melhoramento genético. Viçosa: UFV, 1997. 390p.

EUCLYDES, P.H., FERREIRA, P.A., RUBERT, O.A.V., Regionalização da Vazão Máxima, Mínima e Média de Longo Período e da Curva de Permanência para a Bacia do Rio Doce, Minas Gerais. In: Tecnologia para elaboração de projetos hidroagrícolas em bacias hidrográficas para o Estado de Minas Gerais: Bacia do Rio Doce. Viçosa: Convênio UFV, RURALMINAS, 1996. p.1-96. (Boletim técnico, 3).

HOSKING, J.R.M., WALLIS, J.R. Regional Frequency Analysis: An Approach Based on L-Moments, 224p. Cambridge University Press, Cambridge, Reino Unido, 1997.

LIBERATO, J.R. Aplicações de técnicas de análise multivariada em fitopatologia. Viçosa: UFV, 1995. Dissertação (Mestrado) - Universidade Federal de Viçosa. 144p.

PINHEIRO, M.M.G.; NAGHETTINI, M. Análise regional da freqüência e distribuição temporal das tempestades na Região Metropolitana de Belo Horizonte - RMBH, Revista Brasileira de Recursos Hídricos, v.3, n.4, p. 73 87, 1998.

\section{Regional Analysis of the Storms for Rio Doce Watershed}

\section{ABSTRACT}

This paper presents a methodology for the regional frequency analysis of storms, which was applied to the Doce river watershed. The historical rainfall series for 31 locations were analyzed to identify the homogeneous regions. The moment-L method was used to estimate the parameters of the probability distributions with a better adjustment to average maximum rainfall intensity.

With the average maximum rainfall intensity values corresponding to the different return periods and durations studied, obtained after adjusting the probability distribution with better fit to the observed data, the parameters of the non-linear model were obtained and the intensity-duration-frequency equations were defined for each of the five bomogenous regions..

The residue distributions for the intensity-duration-frequency equations individualized for durations of 10 to 60 minutes and of 60 to 1440 minutes, presented a better performance than for the equation obtained for the period of 10 to 1440 minutes.

The method presented allows estimating the rainfall intensityduration-frequency for a specific duration and return period anywhere 
Análise Regional de Chuvas Intensas para a Bacia do Rio Doce

in the watershed; it is only necessary to know the annual average rainfall.

Key-words: homogeneous areas, probability distribution and non-linear model. 